\title{
Review on Arbuscular Mycorrhizal Fungi: An Approach to Overcome Drought Adversities in Plants
}

\author{
Zaffar Mahdi Dar ${ }^{1 *}$, Amjad Masood ${ }^{2}$, Malik Asif $^{1}$ and Mushtaq Ahamd Malik ${ }^{1}$ \\ ${ }^{1}$ Division of Basic Sciences, Faculty of Agriculture SKUAST-K Kashmir J\&K, India \\ ${ }^{2}$ Division of Agronomy Faculty of Agriculture SKUAST-K Kashmir J\&K, India
}

*Corresponding author

\begin{tabular}{|l|}
\hline Ke y w o r d s \\
Arbuscular \\
mycorhizzae, \\
Drought, Fungi, \\
Tolerance
\end{tabular}

\section{A B S T R A C T}

The ever changing environmental conditions have been faced by plants through evolutionary time, among which drought is considered as the most common having an adverse effect on growth and development of the plants which is one of the major constraints on plant productivity worldwide and is expected to increase with climatic changes. The symbiotic relationship between arbuscular mycorrhizal (AM) fungi and the roots of higher plants is widespread in nature. Several ecophysiological studies have demonstrated that AM symbiosis is a key component in helping plants to cope with water stress and in increasing drought resistance by bringing about various changes in the host plant at the morphological level like alterations in root morphology to form a direct pathway of water uptake by extra radical hyphae. Whereas, the physiological mechanisms promoting enhanced drought tolerance of the host plant by AM inoculation include increased photosynthetic rate and nutrient mobilization followed by their rapid uptake under drought conditions. The biochemical mechanisms promoting drought tolerance in AM inoculated plants include accumulation of osmoprotectants like proline, sugars and trehalose and a rapid enhancement in the concentration of different enzymatic and nonenzymatic antioxidants which reduce the risk of free radical attack on the plant cell membrane which has been evidenced in terms of reduced electrolyte leakage and malondialdehyde (MDA) content of drought stressed plant tissues in various studies.

\section{Introduction}

Crop plants are exposed to several environmental stresses, all affecting plant growth and development, which consequently hampers the productivity of crop plants (Farooq et al., 2011).

Drought is considered the single most devastating environmental stress, which decreases crop productivity more than any other environmental stress (Lambers et al., 2008). A continuous shortfall in precipitation coupled with higher evapotranspiration demand leads to agricultural drought (Mishra and Cherkauer, 2010) which is defined as the lack of ample moisture required for normal plant growth and development to complete the life cycle. In plants, a series of biochemical, physiological and morphological injuries occur due to water stress depending on the factors like duration of exposure of 
plants to the drought, genetic resistance and stage of growth (Gong et al., 2013).

AM fungi are composed of fine, tubular filaments called hyphae. The mass of hyphae that forms the body of the fungus is called the mycelium. There are two major classes of AM fungi: ectotrophic mycorrhizae and vesicular arbuscular mycorrhizae. Ectotrophic AM fungi typically show a thick sheath, or "mantle," of fungal mycelium around the roots, and some of the mycelium penetrates between the cortical cells. The cortical cells themselves are not penetrated by the fungal hyphae but instead are surrounded by a network of hyphae called the hartig net. The capacity of the root system to absorb nutrients is improved by the presence of external fungal hyphae that are much finer than plant roots and can reach beyond the areas of nutrientdepleted soil near the roots (Clarkson, 1985). Ectotrophic AM fungi infect exclusively tree species, including gymnosperms and woody angiosperms. Unlike the ectotrophic AM fungi, vesicular AM fungi do not produce a compact mantle of fungal mycelium around the root. Instead, the hyphae grow in a less dense arrangement, both within the root itself and extending outward from the root into the surrounding soil. After entering the root through either the epidermis or a root hair, the hyphae not only extend through the regions between cells but also penetrate individual cells of the cortex. Within the cells, the hyphae can form oval structures called vesicles and branched structures called arbuscules. The arbuscules appear to be sites of nutrient transfer between the fungus and the host plant. The association of AM fungi with plant roots facilitates the uptake of phosphorus and trace metals such as zinc and copper. By extending beyond the depletion zone for phosphorus around the root, the external mycelium improves phosphorus absorption. Calculations show that a root associated with AM fungi can transport phosphate at a rate more than four times higher than that of a root not associated with AM fungi (Nye and Tinker, 1977). Plants native to arid and semi-arid ecosystems have their roots highly colonised with AM FUNGI, which indicates the significance of AM symbiosis for performance under scarcity of water.

Keeping in view the changing environmental conditions and the increased demand for food and fibre under such conditions, tremendous efforts are being done to satisfy the demands placed on agriculture for food and fibre supply. In order to meet the challenge, a wide variety of efforts focusing on agro ecosystem and soil biological system as a whole is required to understand the stability of process. Keeping in view the concept of sustainability, a vehicle for sustainable agriculture has been hiding secretly for decades in the rhizosphere in the form of plant growth enhancing microbes (Navnita et al., 2015). AM fungi is one of the ancient, diverse and beneficial groups of such soil microbes which other than various anti-stress mechanisms released by the plants during stress can be used to alleviate the drought adversities.

Hence in the following sections, efforts have been made to appraise the role and underlying major mechanisms of AM fungi in plant tolerance to drought stress.

\section{Effect of AM fungi on various features promoting drought tolerance}

\section{Effect of AM fungi on tissue water potential}

Maintenance of tissue water status is an important aspect for improved metabolic activities of the tissues under stressful environment. Stress tolerant plants through a number of strategies could maintain high tissue water status and thereby stress effect is 
not felt by the plants. Therefore, maintenance of tissue water status achieved by different mechanisms like increased water uptake and osmoregulation (discussed below) has lot of relevance for improved growth and productivity of the plants under stressful environments.

\section{Effect of AM fungi on water uptake}

AM fungus plays an important role in water economy and is able to endure much drier soil conditions than most plant life; therefore, plants with a healthy root mycorrhizal population benefit and show better survival under conditions of water stress. Allen and Boosalis (1983) demonstrated how plants treated with AM fungus have a greater tolerance for continued drought. Non mycorrhizal wheat plants and mycorrhizal plants were watered to soil saturation and then allowed to continue transpiring as the soil dried. The stomata of the non mycorrhizal plants began closing and were totally closed after 4 days, but stomata in the mycorrhizal plants did not begin to close as soon and were still transpiring after 6 to 7 days. The results of this study reveal that mycelium maintained the water uptake even under drought conditions by entering deeper into the soil in search of water. A recent finding indicates that AM fungus play a key role in modifications of root hairs consequently helping the plants to overcome the drought (Li et al., 2014). AM fungi expand the roots by adding their own expansive network of absorbing strands to mine the soil for water and the dissolved minerals carried therein may also alter the water relations and the drought tolerance of the plant. Allen et al., (1981a) documented that organisms under water stress conditions exhibited resistance to water transport, but this resistance was decreased by up to $90 \%$ when mycorrhizae were introduced. It has been found that AM fungus improves the hydraulic conductivity and water uptake of roots hence providing a low resistance pathway for radial flow of water across cortex, and contributing towards better water uptake by the plants (Kothari et al., 1990). Furthermore, AM fungus may also regulate the hydraulic properties of plants through regulation of plasma membrane intrinsic proteins in combination with phytohormones (Ruiz-Lozano et al., 2009).

AM fungi mycelia could also improve soil water holding capacity through enhanced soil aggregation (Auge, 2001). Improved soil structure generally improves soil moisture retention properties and as a result, mycorrhizal soil may hold more water at a given soil water potential than non mycorrhizal soil and thus mycorrhizal plants will have access to a larger reservoir of soil water. In association with symbiotic AM fungi, plants could explore larger volumes of soil to absorb water and nutrients thereby impart stress tolerance to the plants (Smith et al., 2009). Tinker (1984) while summarising the effects of VAM on increased water uptake indicated that the probable reason for increased water uptake are as the better distribution of absorbing hyphal network, greater surface area and better extension rate, altered soil rhizosphere properties, uptake kinetics, faster water extraction from reduced water potential soils and hence faster recovery from the water stress. Hyphae may also bridge the gap between soil and root that occurs when soil and root shrink away from each other when dry.

\section{Effect of AM fungi on osmotic adjustment}

Under the condition of water scarcity, water potential of the root cells may increase as a result of less stomatal conductance and more diffusive resistance to carbon dioxide. Therefore, water potential of the root cells is required to be reduced in order to maintain uptake of water from the soil, which in turn is 
achieved by accumulation of different solutes in the cells called as osmoregulation (Munns, 1988).

Osmotic adjustment is one of the important drought adaptive traits, which has lot of relevance in maintaining the tissue water status in plants grown under stressful conditions. Higher osmotic adjustment capacity is a characteristic feature of drought tolerance (Auge, 2001) as it allows the cell to maintain turgor and turgor dependent processes like stomatal opening, cellular expansion, growth, photosynthesis as well as keeping the water potential gradient favourable for water entry into the plant root (Ruiz-Lozano, 2003). When plants are subjected to stress treatment, they produce significantly high amount of several osmolytes and most notable among them are proline, sugars and trehalose whose concentration further increases in $\mathrm{AM}$ inoculated plants subjected to drought stress. Kaya et al., (2009) have reported that the maize plants in association with AM accumulated significantly high amount of proline under salt stress conditions. Similarly in bell pepper, the proline content was found to be increased 3 fold in leaves and 2 fold in roots that suggest the role of AM in enhancing proline accumulation in different tissues facilitating plants to maintain tissue water status (Beltrano et al., 2013). Zhu et al., (2011a) have noticed around 56\% increase in proline level with improved osmotic adjustment in leaves of mycorrhizal plants than non mycorrhizal plants of maize. There is a positive correlation between proline accumulation and AM induced drought tolerance (Azcon et al., 1996). All these studies indicate the relevance of AM in facilitating the plants to go for osmotic adjustment more quickly for maintaining better water relations of the plant tissues. Due to improved osmotic adjustment, the tissue water status was found to be higher in fungal associated plants and therefore, these plants showed greater membrane stability under stress leading to reduced electrolytes leakage. This improvement in membrane stability has also been attributed to increased phosphorus uptake by AM inoculation and changes brought about in membrane phospholipids levels and also in permeability properties (Evelin et al., 2011). During the period of inhibited growth, proline serves as nitrogen and energy source besides reducing the cell water potential (Kala and Godara, 2011). Among osmotic solutes, sugars are equally important as they play an important role in stabilising cell turgor pressure. AM symbiosis increases plant growth and photosynthetic rate which in turn causes increased transport and building up of carbohydrates in cells which act as excellent osmoprotectants to lower osmotic potential (Khalvati et al., 2005). Studies have shown an increase in sugars levels in AM plants exposed to drought in Cyclobalanpsis glauca (Zhang et al., 2014) and in Poncirus trifoliata (Qiangsheng et al., 2006). High sugar content of Poncirus trifoliata confirms a high natural physiological metabolism of AM plants under water stress and well watered conditions leading to accumulation of carbohydrates resulting in decrease of osmotic potential of host cells.

Recently it has been recognized that AM inoculation may also increase drought tolerance of nodulated legumes such as acacia, common bean, lotus, and medicago, through the biosynthesis of trehalose (Lopez et al., 2008). Trehalose, a nonreducing disaccharide, has been found to play a physiological role as drought stress protectant. In the nodules, this disaccharide is synthesized by the bacteroid, and its accumulation depends on the rhizobial strain, legume genotype and environmental conditions. Garcia et al., (2005) and Schubert et al., (1992) have reported that trehalose 
occurs in plants colonized by symbiotic organisms like AM and rhizobia. The accumulation of trehalose by different organisms (bacteria, yeast, fungi, nematodes, etc.) has been related to survival under different environmental stresses, like osmotic, low $\mathrm{pH}$, high and low temperatures and dehydration (Mahmud, 2009). It was reported that under well-watered conditions, there is a basal amount of trehalose in the nodules, having a negative correlation with the biological nitrogen fixation, but under drought conditions, nodule trehalose levels increase significantly and may contribute to maintain biological nitrogen fixation under these circumstances and improve the plant tolerance to drought. Trehalose accumulation in an organism protects the cells by stabilizing cell structures and enables protein to maintain its native confirmation under drought stress. However, the role of trehalose induced by AM fungi in imparting drought tolerance in crop plants needs more investigation.

\section{Effect of AM fungi on antioxidant level}

When plants are subjected to stress, reactive oxygen species (ROS) such as singlet oxygen, superoxides, hydrogen peroxide, and hydroxyl radicals are generated in large quantity causing oxidative damage to the plants. ROS are toxic molecules capable of causing oxidative damage to the lipids, DNA and proteins (Miller et al., 2010). If these molecules are not managed properly, they cause significant damage to the membranes and cause catastrophic effects on cell metabolism. Therefore, efficient quenching of ROS is very crucial for survival and cell metabolism under stress conditions. Stress tolerant plants could manage the ROS through higher activity of antioxidant system (Zhu et al., 2011a). Oxidative stress occurs when the antioxidant defence system is overloaded and is unable to maintain an adequate cellular redox balance. The antioxidant system includes both enzymatic (e.g., superoxide dismutases, ascorbate peroxidases, and catalases) and non-enzymatic molecules (e.g., glutathione, flavonoids, carotenoids, and tocopherols) (Mittler, 2002).

Earlier studies also suggest that the mycorrhizal association helps the plants to overcome the oxidative stress damage and hence, the plants could continue to grow and produce without much of yield penalty under stress conditions. The amelioration of stress resistance by AM symbiosis is often related to the enhancement of antioxidant levels or activities in plants (Baslam and Goicoechea, 2012 and Ruiz-Lozano, 2003) where, they convert the toxic molecules into non-toxic or less toxic molecules.

The level of antioxidant enzymes such as superoxide dismutase, catalase and peroxidase was found to be significantly high in mycorrhizal plants than non mycorrhizal plants when grown under stressful environment (Khalafallah and Abo Ghalia, 2008) hence decreasing the melondialdehyde (MDA) content and plasma membrane conductivity (Baozhong et al., 2010). In drought stressed lettuce plants, AM increased the activity of superoxide dismutase in shoot by $93 \%$ (Ruiz Lozano et al., 1996). The reactive oxygen species generated under stressful condition were quenched efficiently by the action of anti-oxidative enzymes and therefore, the level of reactive oxygen species was found to be significantly low in AM inoculated plants.

Besides antioxidant enzymes, the nonenzymatic system also helped to reduce the level of reactive oxygen species in plants. For example, in the flowering plant cyclamen, higher levels of ascorbic acid and polyphenols besides ascorbate peroxidise and superoxide dismutase were noticed in leaves, roots and tubers of stressed plants suggesting the role of 
non-antioxidant systems to deal with oxidative stress (Maya and Matsubara, 2013). Ruiz Sánchez et al., (2010) found that AM symbiosis ameliorated the response of plants to drought by improving photosynthetic performance but mainly through the accumulation of the antioxidant compound glutathione, which was concomitant with a reduction in oxidative damage to membrane lipids and to low cellular levels of hydrogen peroxide. In maize, Zhu et al., (2011b) have reported low melondialdehyde content in mycorrhizal plants under drought stress which was $17.50 \%$ lower than that of 365 non mycorrhizal plants. In several systems, melondialdehyde has been shown to damage the membranes and disrupting the cell metabolism. Therefore, low level of melondialdehyde observed in maize plants associated with AM fungi is an indication of positive effect of AM fungi on reducing the reactive oxygen species level in plants. These results perhaps support the argument that the mycorrhizal plants could tolerate the stress effects through reduced reactive oxygen species levels under stress conditions.

\section{Effect of AM fungi on photosynthetic rate}

Photosynthesis is generally affected when plants are subjected to stressful environment. Change in leaf orientation, leaf area, stomatal closure, reduced chlorophyll content (Anjum et al., 2003b), decrease in leaf expansion, impaired photosynthetic machinery (Fu J. and Huang, 2001), diminished activities of Calvin cycle enzymes, premature leaf senescence due to drought stress are the possible reasons leading to reduction in photosynthetic rate and food production in stressed plants (Wahid and Rasul, 2005 and Monakhova and Chernyadèv, 2002). When stomatal and non stomatal limitations to photosynthesis are compared, the former can be quite small. This implies that other processes besides $\mathrm{CO}_{2}$ uptake are being damaged due to drought stress. The role of drought induced stomatal closure which limits $\mathrm{CO}_{2}$ uptake by leaves, is very important. In such events, restricted $\mathrm{CO}_{2}$ availability could possibly lead to increased susceptibility to photo damage (Cornic and Massacci, 1996).

Mycorrhizal association has been shown to increase the carbon fixation abilities of the plants. In a number of systems, higher photosynthetic rates have been reported when the plants are in association with AM fungi. For instance, in black locust, Yang et al., (2014) observed high stomatal conductance, high transpiration rates and high photosynthetic rates with reduced internal $\mathrm{CO}_{2}$ concentration in fungal colonized plants than the non-colonized plants. Such higher photosynthetic rate as a consequence of fungal association has also been reported by Zhu et al., (2012). They observed high photosynthetic and transpiration rates in AM fungi colonized plants of maize than in noncolonized plants both under control and drought stress conditions. In these plants, the stomatal conductance was found to be significantly higher indicating the ability of the mycorrhizal plants to keep the stomata wide open to facilitate the exchange of gases both for transpiration and for photosynthesis (Subramanian et al., 1995).

Higher photosynthetic rate observed in most AM inoculated plants is attributed to enhanced water uptake and maintenance of high tissue water status followed by high WUE in these plants. Uptake and movement of sufficient water to the evaporative surface helped the plants to maintain guard cell turgidity leading to opening of stomata for a gaseous exchange process (Nelsen and Safir, 1982). Through the opened stomata, more $\mathrm{CO}_{2}$ enters the plants thereby the photosynthesis is improved in such plants (Zhu et al., 2011b). As the AM inoculated plants has the ability to mine water from soil, 
it could supply the water requirement of the evaporative surface and thus, the transpiration rate was also found to be significantly higher in fungal colonized plants (Roumet et al., 2006). Higher rates of photosynthesis in AM inoculated plants could also be attributed to higher chlorophyll content of the leaves. The AM inoculated plants have shown to have higher chlorophyll content compared to non AM plants. This observation has been made by several workers in different systems (Auge, 2001 and Colla et al., 2008). In pepper for instance, the chlorophyll content was shown to be increased by $15-25 \%$ to indicate the relevance of mycorrhizal association in increasing the pigment content in the leaves (Beltrano et al., 2013). It has been well recognised that chlorophyll concentration is related to photosynthetic rate and chlorophyll fluorescence. Thus, in AM inoculated plants an increased rate of chlorophyll has been associated with the increased rate of photosynthesis or with higher magnesium and nitrogen which are major constituents of chlorophyll (Mathur and Vyas, 1995). Meanwhile, application of AM fungus helps the plants to overcome photo destruction and photoinhibition of pigments under the conditions of water stress by increasing the content of carotenoids, as they help in protection of photosynthetic apparatus against the harm caused by single oxygen. Therefore, quenching and deactivation of excited triplet state of chlorophyll can be brought about by carotenoids (Foyer and Harbinson, 1994) and therefore it is clear that the fungal association is indeed beneficial to plants as it protects the plants from stress effects.

Water is undisputedly the major factor for the declining food production in many parts of the world, particularly in the arid and semiarid regions. Consequently, the world is now being challenged to produce "more crop per drop" of water. Therefore, in recent years there are increasing number of studies to understand the mechanism by which plants alleviate drought stress, and AM fungi seems to be an excellent alternative to serve the purpose. However, the mechanism by which AM fungi promotes drought tolerance is still to be understood well. For example we have a little knowledge about the increased activities of the antioxidants in AM inoculated plants under drought conditions. So the need of the hour is to understand the mechanism so that a perfect plant-AM fungi combination can be formulated for better use of the natural resources, particularly the water, in arid and semi-arid regions of the world.

\section{References}

Allen, M. F., Sexton, J. C., Moore, T. S. Jr. and Christensen, M.1981a. Influence of phosphate source on vesicular-arbuscular mycorrhizae of Bouteloua gracilis. New Phytologist. 87: 687-694.

Allen, M. F. and Boosalis, M. G. 1983. Effects of two species of VA mycorrhizal fungi on drought tolerance of winter wheat. New Phytologist. 93: 67-76.

Anjum, F., Yaseen, M., Rasul, E., Wahid, A. and Anjum, S., 2003b. Water stress in barley (Hordeum vulgare L.). II. Effect on chemical composition and chlorophyll contents. Pakistan J. Agric. Sci. 40: 45-49

Auge, R. M. 2001. Water relations, drought and vesicular arbuscular mycorrhizal symbiosis. Mycorrhiza. 11: 3-42.

Azcon, R., Gomez, M., Tobar, R., 1996. Physiological and nutritional responses by Lactuca sativa L. to nitrogen sources and mycorrhizal fungi under drought conditions. Biology and Fertility of Soils. 22: $155-161$.

Baozhong, Y., Wang, Y., Liu, P., Hu, J and Zhen, W. 2010. Effects of vesicular arbuscular mycorrhiza on the protective system in strawberry leaves under drought stress. Frontiers of Agriculture in China. 4: 165169.

Barzana, G., Aroca, R., Bienert, G. P., Chaumont, F. and Ruiz Lozano, J. M. 2014. New insights into the regulation of aquaporins by 
the arbuscular mycorrhizal symbiosis in maize plants under drought stress and possible implications for plant performance. Molecular Plant Microbe Interaction. 27: 349-363.

Baslam, M. and Goicoechea, N. 2012. Water deficit improved the capacity of arbuscular mycorrhizal fungi (AM FUNGI) for inducing the accumulation of antioxidant compounds in lettuce leaves. Mycorrhiza. 22:347-359.

Beltrano, J., Ruscitti, M., Arango, M. C. and Ronco, M. 2013. Effects of arbuscular mycorrhiza inoculation on plant growth, biological and physiological parameters and mineral nutrition in pepper grown under different salinity and P levels. Journal of Soil Science and Plant Nutrition. 13: 123141.

Clarkson, D. T. 1985. Factors affecting mineral nutrient acquisition by plants. Annual Review of Plant Physiology. 36: 77-115.

Colla, G., Rouphael, Y., Cardarelli, M., Tullio, M., Rivera, C. M. and Rea, E. 2008. Alleviation of salt stress by arbuscular mycorrhizal in zucchini plants grown at low and high phosphorus concentration. Biology and Fertility of Soils. 44: 501-509.

Cornic, G. and Massacci A. 1996. Leaf photosynthesis under drought stress. In: Baker NR, ed. Photosynthesis and the environment. New York: Kluwer Academic Publishers, 347-366.

Evelin, H., Giri, B. and Kapoor, R. 2011. Contribution of Glomus intraradices inoculation to nutrient acquisition and mitigation of ionic imbalance in $\mathrm{NaCl}-$ stressed Trigonella foenum-graecum. Mycorrhiza. 1-15.

Farooq, M., H. Bramley, J.A., Palta, and Siddique, K.H.M. 2011. Heat stress in wheat during reproductive and grain-filling phases. Critical Review in Plant Sciences. 30: 491507.

Foyer, C.H. and J. Harbinson, 1994. Oxygen metabolism and the regulation of photosynthetic electron transport. In: Foyer, C.H. and Mullineausx, P. M. (Ed s). "Causes of photooxidative stress and amelioration of defense system in plants". CRC Press, Boca Raton P, 1-14.
Fu, J. and Huang, B. 2001. Involvement of antioxidants and lipid peroxidation in the adaptation of two cool-season grasses to localized drought stress. Environmental and Experimental Botany. 45: 105-114.

García, N. A., Iribarne, C., López, M., Herrera Cervera, J. A. and Lluch, C. 2005. Physiological implications of trehalase from Phaseolus vulgaris root nodules: partial purification and characterization. Plant Physiology and Biochemistry. 43: 355-361.

Gong, B., Wen, D., Vanden Langenberg, K., Wei, M., Yang, F., Shi, Q. and Wang, X. 2013. Comparative effects of $\mathrm{NaCl}$ and $\mathrm{NaHCO}_{3}$ stress on photosynthetic parameters, nutrient metabolism, and the antioxidant system in tomato leaves. Scientia Horticulture. 157: 1-12.

Kala, S. and Godara, A. K. 2011. Effect of moisture stress on leaf total proteins, proline and free amino acid content in commercial cultivars of Ziziphus mauritiana. Journal of Scientific Research. 55:65- 69.

Kaya, C., Ashraf, M., Sonmez, O., Aydemir, S., Tuna, A.L. and Cullu, M.A. 2009. The influence of arbuscular mycorrhizal colonisation on key growth parameters and fruit yield of pepper plants grown at high salinity. Scientia Horticulture. 121: 1- 6.

Khalafallah, A. A. and Abo Ghalia, H. H. 2008. Effect of arbuscular mycorrhizal fungi on the metabolic products and activity of antioxidant system in wheat plants subjected to short -term water stress, followed by recovery at different growth stages. Journal of Applied Science and Research. 4: 559-569.

Khalvati, M. A., Hu, Y., Mozafar, A. and Schmidhalter U. 2005. Quantification of water uptake by arbuscular mycorrhizal hyphae and its significance for leaf growth, water relations, and gas exchange of barley subjected to drought stress. Plant Biology. 7(6):706-12.

Kothari, S. K., Marschner, H. and George E. 1990. Effect of VA mycorrhizal fungi and rhizosphere microorganisms on root and shoot morphology, growth, and water 
relations of maize. New phytologist. 116: 303-311.

Lambers, H., Chapin, F. and Pons, T. 2008. Plant physiological ecology. Springer, New York, p 540

Li, C., Yue, J., Wu, X., Xu, C., and Yu, J. (2014). An ABA-responsive DRE-binding protein gene from Setaria italica, SiARDP, the target gene of SiAREB, plays a critical role under drought stress. Journal of Experimental Botany. 65: 5415-5427.

Lopez, M., Tejera, N. A., Iribarne, C., Lluch, C., and Herrera-Cervera, J. 2008. Trehalose and trehalase in root nodulae of Medicago truncatula and Phaseolus vulgaris in response to salt stress. Physiologia Plantarum. 134:575-582.

Mahmud, S. A., Nagahisa, K., Hirasawa, T., Yoshikawa, K., Ashitani, K., Shimizu, H. 2009. Effect of trehalose accumulation on response to saline stress in Saccharomyces cerevisiae. Yeast. 26(1):17-30.

Mathur, N. and Vyas, A.1995. Influence of VA mycorrhizae on net photosynthesis and transpiration of Ziziphus mauritiana. Journal of Plant Physiology. 147: 328-330.

Maya, M. A. and Matsubara, Y. 2013. Influence of arbuscular mycorrhiza on the growth and antioxidative activity in cyclamen under heat stress. Mycorrhiza. 23: 381-390.

Miller, G., Suzuki, N., Ciftci-Yilmaz, S., and Mittler, R. 2010. Reactive oxygen species homeostasis and signalling during drought and salinity stresses. Plant Cell Environment. 33: 453-467.

Mishra, V. and Cherkauer, K. A. 2010. Retrospective droughts in the crop growing season: Implications to corn and soybean yield in the midwestern united states. Agriculture and Forest Meteorology. 150: 1030-1045.

Mittler, R. 2002. Oxidative stress, antioxidants and stress tolerance. Trends in Plant Sciences. 7: 405-410.

Monakhova, O.F. and Chernyadèv, I. I. 2002. Protective role of kartolin-4 in wheat plants exposed to soil drought. Applied Biochemistry and Microbiolgy. 38: 373380.

Munns, R. 1988. Effect of high external $\mathrm{NaCl}$ concentrations on ion transport within the shoot of Lupinus albus. I. Ions in xylem sap. Plant, Cell and Environment. 11: 283289.

Navnita, S., Kuldeep, Y., Jagbeer, C., Neetu, B. and Ashok, A. 2015. Arbuscular Mycorrhizal Symbiosis and Water Stress: A Critical Review. Pertanika Journal of Tropical Agriculture Sciences. 38 (4): 427 453.

Nelsen, C. E. and Safir, G. R. 1982. The water relations of well watered, mycorrhizal and non mycorrhizal onion plants. Journal of American Society of Horticultural Science. 107: $271-274$.

Nye, P.H. and Tinker, P.B. 1977. Solute movement in the soil-root system. Blackwell Scientific Publ., Oxford, England

Ocon, A., Hampp, R. and Requena, N. 2007. Trehalose turnover during abiotic stress in arbuscular mycorrhizal fungi. New Phytologist. 174: 879-891.

Qiangsheng, W. U. and Renxue, X. I. A. 2006. Effects of arbuscular mycorrhizal fungi on leaf solutes Arbuscular Mycorrhizal Symbiosis and Water Stress: Frontier for China. 3: 312-317.

Roumet, C., Urcelay, C. and Díaz, S. (2006). Suites of root traits differ between annual and perennial species growing in the field. New Phytologist. 170: 357-68.

Ruiz Lozano, J. M. 2003. Arbuscular mycorrhizal symbiosis and alleviation of osmotic stress. New perspectives for molecular studies. Mycorrhiza. 13: 309-317.

Ruiz-Lozano, J. M., Alguacil, M. M., Bárzana, G., Vernieri, P. and Aroca, R. 2009. Exogenous ABA accentuates the differences in root hydraulic properties between mycorrhizal and non mycorrhizal maize plants through regulation of PIP aquaporins. Plant Molecular Biology. 70: 565-579.

Ruiz-Lozano, J. M., Azcón, R. and Gómez M. 1996. Alleviation of salt stress by arbuscular mycorrhizal Glomusspecies in Lactuca sativa plants. Physiologia Plantarum. 98: 767-772.

Ruiz-Sanchez, M., Aroca, R., Munoz, Y., Armada, E., Polon, R. and Ruiz-Lozano, J. M. 2010. The arbuscular mycorrhizal symbiosis enhances the photosynthetic efficiency and the antioxidative response of 
rice plants subjected to drought stress. Journal of Plant Physiology. 167:862-869.

Schubert, A., Wyss, P. and Wiemken A. 1992. Occurrence of trehalose in vesiculararbuscular mycorrhizal fungi and in mycorrhizal roots. Journal of Plant Physiology. 140: 41-45.

Smith, F. A., Grace, E. J. and Smith, S. E. 2009. More than a carbon economy: nutrient trade and ecological sustainability in facultative arbuscular mycorrhizal symbioses. New Phytologist. 182:347-358.

Subramanian, K. S., Charest, C., Dwyer, L. M. and Hamilton, R. I. 1995. Arbuscular mycorrhiza and water relations in maize under drought stress at tasselling. New Phytologist. 129: 643-650.

Tinker, P. B. 1984. The role of microorganisms in mediating and facilitating the uptake of plant nutrients from soil. Plant and Soil. 76: 77-91.

Wahid, A. and Rasul, E. 2005. Photosynthesis in leaf, stem, flower and fruit, in: Pessarakli M. (Ed.), Handbook of Photosynthesis, 2nd ed., CRC Press, Florida, pp. 479-497

Yang, Y., Tang, M., Sulpice, R., Chen, H., Tian, S. and Ban, Y. 2014. Arbuscular mycorrhizal fungi alter fractal dimension characteristics of Robinia pseudoacacia L. seedlings through regulating plant growth, leaf water status, photosynthesis, and nutrient concentration under drought stress. Journal of Plant Growth Regulators. 33: 612-625.

Zhang, J. Y., Cruz, D., Torres-Jerez, I., Kang, Y., Allen, S. N., Huhman, D. V., Tang, Y., Murray, J., Sumner, L. W. and Udvardi, M. K. (2014). Global reprogramming of transcription and metabolism in Medicago truncatula during progressive drought and after rewatering. Plant Cell Environment. $37: 2553-76$.

Zhu, X. C., Song, F. B., Liu, S. Q., Liu, T. D and Zhou, X. (2012). Arbuscular mycorrhizae improve photosynthesis and water status of Zea mays L. under drought stress. Plant Soil and Environment. 58(4): 186-191.

Zhu, X., Song, F. and Liu, S. 2011a. Arbuscular mycorrhiza impacts on drought stress of maize plants by lipid peroxidation, proline content and activity of antioxidant system. Journal of Food, Agriculture and Environment. 9: 583-587.

Zhu, X., Song, F. B., Liu, S., and Liu, T. 2011b. Effects of arbuscular mycorrhizal fungus on photosynthesis and water status of maize under high temperature stress. Plant and Soil. 346: 189-199.

\section{How to cite this article:}

Zaffar Mahdi Dar, Amjad Masood, Malik Asif and Mushtaq Ahamd Malik. 2018. Review on Arbuscular Mycorrhizal Fungi: An Approach to Overcome Drought Adversities in Plants. Int.J.Curr.Microbiol.App.Sci. 7(03): 1040-1049. doi: https://doi.org/10.20546/ijcmas.2018.703.124 